\title{
Integration durch Usurpation - Thesen zur Radikalisierung der Binnenmarktintegration
}

Eine Serie kontroverser Urteile des Europäischen Gerichtshofs (EuGH) hat jene vor den Kopf gestoßen, die sich von der europäischen Integration sozialpolitische Impulse, mithin die Entstehung eines „europäischen Sozialraums“ erhoffen. Bereits seit den 1960er Jahren usurpierte der EuGH unter Umgehung der politischen Willensbildung Souveränitätsrechte der Mitgliedstaaten. Unlängst haben sich die Übergriffe des EuGH auf die Gestaltungshoheit der EU-Mitglieder radikalisiert. Die jüngsten Akte der Überinterpretation der europäischen Grundfreiheiten sind Kampfansagen an den Bestand unterschiedlicher nationaler Lösungen zum Ausgleich von kapitalistischer Ökonomie und sozialer Demokratie. ${ }^{1}$

\section{1 \\ Einleitung}

Die seit Dezember 2007 ergangenen vier EuGH-Urteile in den Rechtssachen Viking, Laval, Rüffert und Kommission gegen Luxemburg haben eine kontroverse Diskussion nicht nur innerhalb der rechtswissenschaftlichen Fachdisziplin, sondern auch in Politik und Verbänden und namentlich den Gewerkschaften angestoßen. Gewerkschaftsnahe Publikationen wie der Einblick, Mitbestimmung und böckler impuls berichteten mehrfach, namhafte Sozial- und Rechtswissenschaftler und Gewerkschafter der nationalen und der europäischen Ebene nahmen Stellung - mit zum Teil recht unterschiedlichen Stoßrichtungen. Während beispielsweise der Politikwissenschaftler Fritz W. Scharpf in einem viel beachteten Interview mit der Mitbestimmung forderte, dem EuGH den Rechtsgehorsam zu verweigern (Scharpf 2008a), begrüßte der Europäische Gewerkschaftsbund (EGB) in seiner ersten Stellungnahme zum VikingUrteil, dass der EuGH das Streikrecht als allgemeinen Rechtsgrundsatz der Gemeinschaft anerkannt habe - und warnte, „Gewerkschaften und Arbeitnehmer in ganz Europa“ könnten nunmehr „sehr darauf bedacht [sein], ihre jeweiligen nationalen Systeme zu schützen - sodass die Gefahr einer protektionistischen Reaktion besteht".2

Der Beitrag knüpft an diese Debatte an und spitzt sie im Hinblick auf Implikatio- nen einer politikwissenschaftlich orientierten, historisch-institutionalistischen Perspektive $^{3}$ auf die Rechtsfortbildung durch den EuGH zu. Die Urteile zu Viking, Laval, Rüffert und Kommission gegen Luxemburg haben gemein, dass sie die europäischen Grundfreiheiten - konkret: die Dienstleistungs- und die Niederlassungsfreiheit zur Umgestaltung von Elementen mitgliedstaatlichen Arbeitsrechts (Streikrecht, Mindestlöhne) nutzen, die eigentlich, so die herrschende Meinung vor den Urteilen, in den ausschließlichen Kompetenzbereich der Mitgliedstaaten fallen. Da die Urteile und die zugrundeliegenden Fälle bereits in einer Vielzahl von Publikationen dargestellt wurden, werde ich mich nachfolgend auf die vertiefende Diskussion konzentrieren. ${ }^{4}$ Ich werde die vier Urteile zunächst in den Kontext früherer rechtsfortbildender EuGH-Entscheidungen stellen (Abschnitt 2) und darauf aufbauend die mit den Urteilen ergangene zusätzliche Rechtsfortbildung würdigen (Abschnitt 3). Sie besteht darin, dass der EuGH den nationalstaatlichen Spielraum zur Formulierung unterschiedlicher Antworten auf den grundlegenden Konflikt zwischen kapitalistischer Ökonomie und sozialer Demokratie in vorher nicht gekannter Weise einengt und die Bereitschaft ankündigt, weitere solche Einengungen vorzunehmen. Dann werde ich mich den Reaktionen auf die Urteile zuwenden. Ich werde Thesen dazu formulieren, warum offensiver Schutz nationaler Rechtsbestände vor politisch nicht legitimierten Übergriffen europäischen Richterrechts - und, damit verbunden, die
1 Der Beitrag ist eine stark gekürzte, um einen zusätzlichen Abschnitt ergänzte und auf die gewerkschaftliche Debatte zugespitzte Fassung eines Discussion-Papers des Max-Planck-Instituts für Gesellschaftsforschung (MPIfG) (Höpner 2008). Mein Dank gilt Florian Rödl und Fritz W. Scharpf für ihre ausführlichen Gutachten sowie weit darüber hinausreichende Hilfestellungen. Des Weiteren danke ich Michael Blauberger, Christian Joerges, Andreas Nölke, Alexander Petring, Susanne K. Schmidt, Daniel Seikel, Benjamin Werner sowie zwei anonymen Gutachtern der WSI-Mitteilungen für wertvolle Hinweise.

2 Vgl. "Stellungnahme des Europäischen Gewerkschaftsbunds zu den Urteilen des EuGH in den

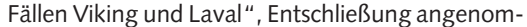
men vom EGB-Exekutivausschuss auf seiner Sitzung am 4. März in Brüssel, S. 3, online: http:// www.etuc.org/IMG/pdf_ResolutionDE-2.pdf.

3 Der "historische Institutionalismus “ analysiert gesellschaftlichen Wandel als sukzessive, sich über lange historische Prozesse vollziehende Reinterpretation von Institutionen (Streeck/Thelen 2005). Es kommt mir hier darauf an, die jüngere Rechtsprechung des EuGH nicht als juristisches Problem "richtig" oder "falsch" urteilender Richter, sondern als Teil eines solchen historischen Prozesses zu verstehen.

4 Für eine Darstellung der Fälle verweise ich auf Höpner (2008, Abschnitt 2). Die nachfolgende Argumentation setzt Vertrautheit mit den konkreten Fällen aber nicht voraus.

Martin Höpner, PD Dr., Politikwissenschaftler, leitet am Kölner Max-Planck-Institut für Gesellschaftsforschung die unabhängige Forschungsgruppe "Europäische Liberalisierungspolitik". Arbeitsschwerpunkte: Spielarten des Kapitalismus, vergleichende Politische Ökonomie, Integrations- und Policy-Forschung. e-mail: hoepner@mpifg.de. 
Definition von Grenzen der europäischen Integration - der gewerkschaftlichen Programmatik nicht leicht fällt (Abschnitt 4). Zudem werde ich eine vorsichtige Einschätzung zu den Erfolgsaussichten der zentralen gewerkschaftlichen Antwort auf die Urteile formulieren (Abschnitt 5). Dabei geht es um den Wunsch nach Einfügung sozialer Grundrechte in das europäische Primärrecht, verbunden mit der Forderung, diese Grundrechte in Abwägungsfragen höher zu gewichten als die Grundfreiheiten (freie Bewegung von Waren, Kapital, Personen und Dienstleistungen).

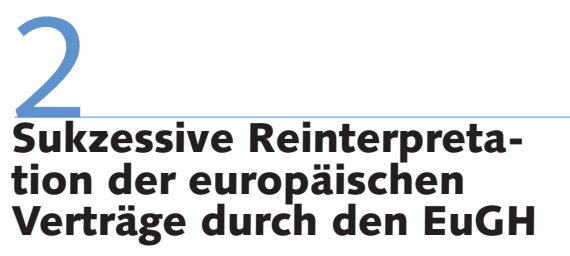

Die europäische Integration beruht auf dem Verhandlungsgeschick visionärer Staatsund Regierungschefs, denen es in einer historischen Abfolge von Vertragsschlüssen gelang, sich auf die Delegation zunehmender Anteile ihrer Kompetenzen an die europäische Ebene zu einigen - so könnte man meinen. Tatsächlich aber beruht ein erheblicher Teil der Integration nicht auf Delegation von unten, sondern auf der richterlichen Reinterpretation der Verträge und der damit einhergehenden Usurpation von Kompetenzen. ${ }^{5}$ Anhand der Urteile zu Viking, Laval, Rüffert und Kommission gegen Luxemburg lässt sich dieser Sachverhalt demonstrieren. Denn fragen wir, auf Basis welcher Grundsätze sie möglich wurden, so werden wir mit Ausnahme der vor fünfzig Jahren in den Römischen Verträgen niedergelegten Grundfreiheiten wenig finden, an dem die Politik aktiv beteiligt war. Die Urteile zehren von Grundsätzen, die ihrerseits durch Richterrecht geschaffen wurden, und markieren selbst einen weiteren Akt der Reinterpretation europäischen Primär- und Sekundärrechts.

\subsection{UNMITTELBARE WIRKUNG DES EUROPARECHTS}

Die Römischen Verträge von 1957 waren auf Grundlage des Völkerrechts geschlossene Verträge zur Förderung der wirtschaftlichen Zusammenarbeit. Völkerrechtliche Verträge entfalten Wirkung zwischen den vertragschließenden Staaten, ohne dass sich daraus Rechte der Wirtschaftssubjekte gegenüber den Staaten ableiten lassen. Bereits in den 1960er Jahren arbeitete der EuGH die unmittelbare Wirkung (auch: Direktwirkung) des Gemeinschaftsrechts heraus, emanzipierte das Europarecht damit vom Völkerrecht und leitete die Konstitutionalisierung der damaligen Europäischen Wirtschaftsgemeinschaft ein. In seinem Urteil zu van Gend \& Loos (C-26/62 vom 5.2.1963), das durch einen Rechtsstreit zwischen einer niederländischen Transportfirma und den dortigen Zollbehörden ausgelöst wurde, urteilte der $\mathrm{EuGH}$, „dass die Gemeinschaft eine neue Rechtsordnung des Völkerrechts darstellt, zu deren Gunsten die Staaten, wenn auch in begrenztem Rahmen, ihre Souveränitätsrechte eingeschränkt haben, eine Rechtsordnung, deren Rechtssubjekte nicht nur die Mitgliedstaaten, sondern auch die Einzelnen sind" (Rn. 10). Das bedeutete im konkreten Fall: Die Transportfirma van Gend \& Loos konnte sich vor den nationalen Gerichten auf die Römischen Verträge berufen und aus ihnen das Recht ableiten, den von den Zollbehörden verlangten überhöhten Einfuhrzoll von $6 \%$ nicht zahlen zu müssen. Der EuGH hatte das Europa der Sechs damit zu einer Rechtsgemeinschaft fortentwickelt, deren Recht unmittelbar auf die Bürger wirkt.

Wie Hitzel-Cassagnes (2004, S. 124f.) im Einzelnen zeigt, erfuhr das Prinzip der unmittelbaren Wirkung des Gemeinschaftsrechts durch nachfolgende Urteile eine mehrfache sukzessive Ausweitung. Im Urteil zur Rechtssache Lütticke (C-57/56 vom 16.6.1966) wurde der Grundsatz der unmittelbaren Wirkung von einer negativen zu einer positiven Verpflichtung bzw. von einer Unterlassungs- zur Handlungspflicht ausgebaut. Im Urteil zur Rechtssache van Duyn (C-41/74 vom 4.12.1974) wurde maßgeblich der Grundsatz der unmittelbaren Wirkung auch des Sekundärrechts formuliert, also der unmittelbaren Wirkung nicht nur der Vertragsbestimmungen, sondern auch der Gemeinschaftsrechtssetzung. In einem weiteren Schritt wurde die unmittelbare Wirkung des europäischen Rechts auch auf EG-Abkommen ausgedehnt (Rechtssache Kupferberg, C-104/81 vom 26.10.1982).

\subsection{VORRANG VOR NATIONALEM RECHT}

Seine Brisanz gewinnt das Prinzip der unmittelbaren Wirkung in Verbindung mit dem ebenfalls bereits in den frühen 1960er Jahren vom EuGH entwickelten Prinzip des Vorrangs des europäischen Rechts vor nationalem Recht. In der konkreten Rechtssache ging es um den Widerstand eines Aktionärs gegen die Verstaatlichung des italienischen Stromwesens. Er argumentierte, das italienische Verstaatlichungsgesetz stünde nicht im Einklang mit den Römischen Verträgen, und beantragte, das nationale Gericht möge die Frage der Vereinbarkeit dem EuGH vorlegen. In den Römischen Verträgen war die Nachrangigkeit des nationalen Rechts gegenüber europäischem Recht nicht festgeschrieben, und folglich argumentierte der italienische Staat, der EuGH habe nicht über die Quasi-Verfassungsmäßigkeit des betroffenen Gesetzes zu entscheiden. Der EuGH aber entschied im Urteil zur Rechtssache Costa/ ENEL (C-6/46 vom 15.7.1964), dass „dem vom Vertrag geschaffenen, somit aus einer autonomen Rechtsquelle fließenden Recht wegen dieser seiner Eigenständigkeit keine wie auch immer gearteten innerstaatlichen Rechtsvorschriften vorgehen können... Die Staaten haben... eine endgültige Beschränkung ihrer Hoheitsrechte bewirkt" (aus den Rn. 12 und 13). Kollidieren also nationale und europäische Normen, dürfen die nationalen nicht angewendet werden. Dieser Umdeutungsschritt des EuGH ist erst vollständig gewürdigt, wenn zusätzlich bedacht wird, dass der EuGH hier zwischen nationalem Primär- und Sekundärrecht nicht unterschied und in einem weiteren Urteil explizit klarstellte, dass der Vorrang jeglichen europäischen Rechts auch für nationales Verfassungsrecht, einschließlich der Grundrechte, gilt (Urteil im Fall Internationale Handelsgesellschaft, C-11/70 vom 17.12.1970). Einige Jahre später urteilten die europäischen Richter, dass den nationalen Verfassungsgerichten bei Kollisionsfällen keine Prüfungsrechte zustehen (Urteil im Fall Simmenthal II, C-106/77 vom 9.3.1978).

\subsection{RECHTSBINDUNG PRIVATER}

Eine weitere, als eigenständige Rechtsfortbildung $\mathrm{zu}$ wertende Entwicklungslinie tritt hinzu: die unmittelbare Verpflichtung Privater durch die Vertragsbestimmungen und die Grundfreiheiten im Be-

\footnotetext{
5 Usurpation ist definiert als Aneignung hoheits-
} staatlicher Befugnisse. 
sonderen. ${ }^{6}$ Der Grundsatz der Verpflichtung Privater durch das europäische Recht wurde erstmals in den Rechtssachen Defrenne (C-43/75 vom 8.4.1976) und Walrave und Koch (C-36/74 vom 12.12.1974) entwickelt. In diesen Urteilen entschied der EuGH, dass der Grundsatz des Verbots geschlechtsspezifischer Entgeltdiskriminierung direkte Wirkung auf eine belgische Fluggesellschaft entfaltet (Defrenne) und dass die Niederlassungsfreiheit auch Verbänden - im konkreten Fall: einem Radsportverband - unmittelbare Pflichten auferlegte (Walrave und Koch). Sukzessive Ausweitungen dieses Grundsatzes erfolgten mit den Urteilen zu Bosman (C-415/93 vom 15.12. 1995) und Angonese (C-281/98 vom 6.6.2000). Diese Linie lässt sich bis zur jüngsten Urteilsserie weiterverfolgen, die mit Viking und Laval Gewerkschaften die Pflicht auferlegt, die Ausübung der Grundfreiheiten nicht durch Streiks zu stören.

Fassen wir bis hier zusammen: In einer Serie „revolutionärer rechtsdogmatischer Schritte" (Haltern 2005, S. 552; Vauchez 2007) hatte der EuGH, ohne Zutun der Mitgliedstaaten, einen völkerrechtlichen Vertrag zu einer Quasi-Verfassung umgedeutet und die Grundsätze des Vorrangs und der unmittelbaren Wirkung auch auf das europäische Sekundärrecht ausgedehnt (Rasmussen 1986). Die Folge, jedenfalls in der (von nationalen Verfassungsgerichten nicht gänzlich akzeptierten $)^{7}$ Interpretation des EuGH: Jede europäische Richtlinie und jede Interpretation einer Richtlinie durch den EuGH bricht nationales Verfassungsrecht. Damit klärt sich ein Umstand, der insbesondere am Urteil zu Laval verstört. Wie kann es sein, dass eine europäische Richtlinie - bei Laval sogar: eine höchst überraschende, der ursprünglichen Intention des Ministerrats mit hoher Wahrscheinlichkeit zuwiderlaufende Interpretation der Entsenderichtlinie - der Ausübung eines nationalen Grundrechts Grenzen setzt, für das die Gemeinschaft nicht zuständig ist? Die Antwort liegt in der Kombination von Vorrangwirkung europäischen Sekundärrechts vor nationalem Grundrecht einerseits und unmittelbarer Drittwirkung der europäischen Grundfreiheiten andererseits.

\subsection{BESCHRÄNKUNGSVERBOTE}

Die Grundsätze der unmittelbaren Wirkung und des Vorrangs potenzieren somit die Durchschlagskraft der in den Römi- schen Verträgen niedergelegten vier Grundfreiheiten: der Warenverkehrsfreiheit, der Freizügigkeit, der Dienstleistungsfreiheit und der Kapitalverkehrsfreiheit. Aber auch die Zielsetzungen dieser Grundfreiheiten selbst wurden durch die Rechtsprechung des EuGH über den Wortlaut der Verträge hinaus radikalisiert, genauer: Der EuGH deutete Diskriminierungs- in Beschränkungsverbote um. Im Vertragstext stellten die Binnenmarktregeln darauf $a b$, dass Wirtschaftsteilnehmer aus anderen Mitgliedsländern auf heimischen Märkten nicht schlechter gestellt werden durften als Inländer. Es musste also ein Diskriminierungstatbestand vorliegen, damit die Handlungen von Staaten oder Wirtschaftsteilnehmern auf Grundlage der Grundfreiheiten sanktioniert werden konnten. Etwa ein Jahrzehnt nach der Durchsetzung von Vorrang und unmittelbarer Wirkung des Europarechts begann der EuGH, seine Rechtsprechung zu den Binnenmarktregeln in Richtung allgemeiner Beschränkungsverbote auszubauen. In der berühmten Dassonville-Entscheidung (C-8/74 vom 11.7.1974) - ein belgischer Großhändler hatte sich dagegen gewehrt, aufwendige Ursprungsbescheinigungen zum Import schottischen Whiskys beschaffen zu müssen -, stellte der EuGH heraus: „Jede Handelsregelung der Mitgliedstaaten, die geeignet ist, den innergemeinschaftlichen Handel unmittelbar oder mittelbar, tatsächlich oder potenziell zu behindern, ist als Maßnahme mit gleicher Wirkung wie eine (im Binnenmarkt nach Art. 28 EGV verbotene, $\mathrm{MH})$ mengenmäßige Beschränkung anzusehen" (Rn. 5).

Die praktische Wirkung dieser Umdeutung war enorm, kam sie doch einer immensen Ausweitung des Anwendungsbereichs europäischen Rechts gleich. Mit diesem qualitativen Schritt wurden Vorrang, Direktwirkung und unmittelbare Drittwirkung zu Instrumenten wirtschaftlicher Liberalisierung, ohne bei deren Schöpfung durch den EuGH als solche angelegt gewesen zu sein. ${ }^{8}$ Potenzielle Hindernisse des transnationalen Warenverkehrs waren nunmehr per se europarechtswidrig, auch wenn sie unterschiedslos und damit diskriminierungsfrei auf einheimische wie ausländische Anbieter angewendet wurden, also unabhängig davon, ob sie ihrer Intention nach protektionistisch waren oder nicht (Büchele 2008, S. 352ff.; Schmidt 2008, S. 106). Freilich war eine Judikatur, die die Freiheit des Warenverkehrs über alles stellt, kaum vorstellbar. In seiner ebenso berühmten Cassis de Dijon-Entscheidung zum Verbot von Einfuhrbeschränkungen für Waren, die in anderen Mitgliedstaaten zugelassen waren (in diesem Fall: Europarechtswidrigkeit der Einfuhrbeschränkung für einen Likör, C-120/78 vom 20.2.1979), formulierte der EuGH das Kriterium für Grenzen des allgemeinen Beschränkungsverbots: „Hemmnisse für den Binnenhandel der Gemeinschaft ... müssen hingenommen werden, soweit diese Bestimmungen notwendig sind, um zwingenden Erfordernissen gerecht zu werden, insbesondere den Erfordernissen einer wirksamen steuerlichen Kontrolle, des Schutzes der öffentlichen Gesundheit, der Lauterkeit des Handelsverkehrs und des Verbraucherschutzes" (Rn. 8). Entscheidend ist hier weniger, dass der EuGH die Möglichkeit von Beschränkungen der Grundfreiheiten bejahte, als vielmehr, dass er solchen Beschränkungen hohe Hürden auferlegte (,zwingende Erfordernisse") und sich selbst, nicht etwa den betroffenen Mitgliedstaaten, das Prüfungsrecht zugestand.

In den nachfolgenden Jahren weitete der EuGH seine Dassonville- und Cassis de Dijon-Rechtsprechung auch auf die anderen Grundfreiheiten aus, und es ist gerade dieses „Durchdeklinieren“ des Beschränkungsverbots auf alle Felder wirtschaftlichen Handelns, das den EuGH in Kernbereiche mitgliedstaatlicher Souveränität eingreifen lässt (vgl. Abschnitt 3). Als Beispiele seien die in den Bereich der Arbeitnehmerfreizügigkeit fallenden Entschei-

6 Für diesen Hinweis sowie Verweise auf die entscheidenden Urteile danke ich Florian Rödl. Mitunter, so bei Hitzel-Cassagnes (2004, S. 124f.), wird die Verpflichtung Privater durch das Europarecht als Fortsetzung der Rechtsprechung zur unmittelbaren Wirkung gedeutet.

7 Bis heute hat beispielsweise das deutsche Bundesverfassungsgericht den Grundsatz des Vorrangs europäischen Rechts vor nationalem Grundrechtsbestand nicht abschließend akzeptiert. In diesem Zusammenhang ist bemerkenswert, dass Artikel I-6 des nicht ratifizierten europäischen Verfassungsentwurfs die erstmalige und unwiderrufliche Festschreibung des Vorrangprinzips in den Verträgen vorgesehen hatte: Europäisches Primär- und Sekundärrecht, so der Text, "haben Vorrang vor dem Recht der Mitgliedstaaten “.

8 Hier besteht Anschlussfähigkeit zu Streeck und Thelens (2005, S. 26ff.) Einsichten in Mechanismen institutionellen Wandels: Institutionen, so auch Rechtsinstitutionen, können dadurch faktischen Wandel erfahren, dass sie bei gleichbleibenden strukturellen Eigenschaften für Zwecke genutzt werden, für die sie ursprünglich nicht gedacht waren (institutional conversion) 
dungen zu Bosman (C-415/93 vom 15.12. 1995) und Graf (C-190/98 vom 27.1.2000) genannt, denen zufolge alle Regeln verboten sind, die geeignet sind, die Freizügigkeit von Arbeitnehmern zu beeinträchtigen, und zwar auch dann, wenn sie unabhängig von der Staatsangehörigkeit der betroffenen Beschäftigten angewendet werden (Frenz 2004, S. 554ff.). Ähnliches gilt für jüngere, sehr umstrittene Urteile zur Niederlassungs- und Kapitalverkehrsfreiheit. Im Ergebnis also sind die Verträge zu Rechtsquellen geworden, die den Wirtschaftsteilnehmern Ansprüche gegenüber ihren Staaten (unmittelbare Wirkung) auf Nichtanwendung (Vorrang des europäischen Rechts) marktbegrenzender Regelungen sowie auf Unterlassung von Handlungen Privater (Drittwirkung) verliehen, soweit die betroffenen Gebietskörperschaften vor dem EuGH nicht nachweisen konnten, dass die Regelungen oder Handlungen zwingenden Gründen des Allgemeininteresses dienten (Cassis-Formel) und verhältnismäßig waren - ob sie nun einen diskriminierenden, also protektionistischen Hintergrund hatten oder nicht (DassonvilleUrteil).

\section{3 \\ Der qualitativ neue Schritt der jüngsten Urteile}

In Abschnitt 5 werde ich zum bisher Dargelegten eine weitere Klasse rechtsfortbildender EuGH-Urteile hinzuaddieren: die Konstruktion europäischer Grundrechte als „allgemeine Rechtsgrundsätze der Gemeinschaft". Aber bereits mit dem bis hier entwickelten Stand der Argumentation sind wir in der Lage, die mit den Urteilen zu Viking, Laval, Rüffert und Kommission gegen Luxemburg vollzogenen Innovationen zu würdigen. Die darin zum Ausdruck kommende „Radikalisierung der Binnenmarktintegration" (Scharpf) baut auf Grundlagen auf, die sich der EuGH bereits in den 1960er bis 1970er Jahren geschaffen hatte. An den Urteilen irritiert, dass sie gesellschaftliche Felder wie das Streik- und Arbeitsrecht tangieren, für die die Organe der EU eigentlich nicht zuständig sind. Im Bereich der europäischen Gesetzgebung wurde diese Nichtzuständigkeit explizit als Sperrklausel in den Vertrag aufgenommen: Die Kompetenz der Gesetzgebungsorgane „gilt nicht für das Arbeitsentgelt, das Koali- tionsrecht, das Streikrecht sowie das Aussperrungsrecht" (aus Art. 137 Abs. 5 EGV). Umstritten blieb stets, was dieser politische Wunsch nach Schutz der nationalen Autonomie für den EuGH bedeutete, konnte er diese Regelungsfelder doch de facto durch Anwendung der oben herausgearbeiteten Instrumente transformieren. Solange er davon Abstand nahm, geschah dies - wie wir heute wissen - als Akt der Selbstzurückhaltung vor Radikalisierungen, die juristisch gleichwohl möglich erschienen.

\subsection{VON DER SUCHE NACH VERSTECKTEM PROTEKTIONISMUS...}

Bereits in früheren Entscheidungen hatte der EuGH Elemente der Arbeits- und Sozialverfassungen auf ihre Verhältnismäßigkeit gegen die europäischen Grundfreiheiten geprüft (vgl. die ausführliche Diskussion bei Rödl 2009, Abschnitt 3). Der Unterschied zur jüngsten Urteilsserie bestand lediglich darin, dass er in der Vergangenheit dabei stets autonomieschonend vorging. Es galt als ausgemacht, dass europäische Grundfreiheiten das Arbeitsrecht nicht umzuorganisieren haben. Unter diesen Bedingungen hatte die Prüfung durch den EuGH, wie Rödl ausführt, vor allem den Charakter einer Missbrauchskontrolle, also der Prüfung auf versteckten Protektionismus. Im Urteil zum Fall Albany (C-67/96 vom 21.9.1999) zum Beispiel bejahte der EuGH den autonomen Regelungsspielraum der niederländischen Sozialpartner, Arbeitnehmern die Mitgliedschaft in einem Betriebsrentenfonds verbindlich vorzuschreiben, obwohl dies wettbewerbsbeschränkende Wirkungen entfalte.

Auch außerhalb des Arbeitsrechts gelegene Formen der Grundrechtsausübung wurden schon vor der jüngsten Urteilsserie gegen die Grundfreiheiten geprüft. Im Fall Schmidberger (C-112/00) hatte der EuGH darüber zu befinden, ob Österreich einer Gruppe von Umweltschützern erlauben durfte, eine zeitweilige Vollblockade der Brenner-Autobahn durchzuführen, obwohl dies den freien Warenverkehr behinderte. Das Gericht stellte heraus, der Grundrechtsschutz - in diesem Fall: die Versammlungsfreiheit - stelle ein Interesse dar, das geeignet sei, eine Einschränkung der europäischen Grundfreiheiten zu rechtfertigen (Rn. 82). Und in seiner Entscheidung zum Fall Omega Spielhallen (C-36/02 vom 14.10.2004) sah der EuGH das Verbot brutaler Spielhallengeräte durch die Stadt
Bonn aus Gründen der öffentlichen Ordnung als mit dem europäischen Recht vereinbar an, obwohl das Verbot eine Beschränkung der Dienstleistungsfreiheit mit sich brachte. Gleichwohl gilt: Der EuGH prüfte, ob zwingende Gründe des Allgemeininteresses vorlagen, die Beschränkungen der Grundfreiheiten rechtfertigten.

\section{2 ...ZUR OKTROYIERTEN HARMONISIERUNG MITGLIED- STAATLICHER RECHTSBESTÄNDE}

Die Innovation der Urteile Viking, Laval, Rüffert und Luxemburg besteht also nicht in der Verhältnismäßigkeitsprüfung selbst, sondern in deren Ergebnis: Die verbleibenden Spielräume der betroffenen Staaten und der Sozialpartner schmelzen auf ein Minimum zusammen. Der EuGH benutzt vorher bereits vorhandene Instrumente für neue Ziele. Was vorher den Charakter einer Missbrauchsprüfung hatte, wird nunmehr zu einem Instrument der Umgestaltung eigentlich allein den Mitgliedstaaten zustehender Rechtsbestände - bis hin zu Arbeitskampfverboten an Gewerkschaften (Anwendung des Beschränkungsverbots auf alle Grundfreiheiten, Radikalisierung der Rechtsprechung zur Drittwirkung). Im Ergebnis stellt der EuGH auch die Grundrechtsausübung unter den grundsätzlichen Vorbehalt der europäischen Grundfreiheiten, ein de facto Schutzbereich dieser Rechtsbestände vor Unterordnung unter die Binnenmarktregeln ist nicht mehr erkennbar.

An dieser Stelle, so denke ich, ist die für den Bestand der „organisierten “ Ökonomien des europäischen Kontinents gefährliche Konstellation deutlich geworden, die durch die lange, politisch ungestörte Serie der Rechtsfortbildung durch europäische Richter entstanden ist. Zwar haben wir es bei den Fällen Viking bis Kommission gegen Luxemburg lediglich mit einer Radikalisierung der Anwendung von Instrumenten zu tun, die schon vorher bereitlagen. Hinsichtlich der praktischen Auswirkungen aber kann der hier getätigte Schritt gar nicht groß genug eingeschätzt werden. Denn die Verknüpfung von Vorrang, Direktwirkung, Beschränkungsverbot und dem Mut, sogar den von Art. 137 Abs. 5 EGV betroffenen Politikfeldern bei der Verhältnismäßigkeitsprüfung de facto keinen besonderen Schutzbereich mehr einzuräumen, ist im Prinzip geeignet, marktkorrigierende Regulierungen der Mitgliedstaa- 
ten bis auf ein Minimum einzuebnen. In diesem Sinne können und müssen die Urteile zu Viking, Laval, Rüffert und Luxemburg als Kampfansagen an die Existenz autonomer Handlungsspielräume zum Ausgleich von kapitalistischer Ökonomie und sozialer Demokratie verstanden werden.

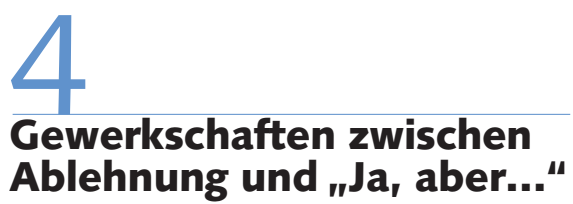

Die Ausdehnung des Anwendungsbereichs der Grundfreiheiten auf neue Sachverhalte verstört, wie ich oben gezeigt habe, hinsichtlich ihrer materiellen Wirkungen (transformative Eingriffe; Output-Dimension) ebenso wie hinsichtlich ihrer legitimatorischen Grundlagen (politisch nicht gedecktes Richterrecht, Input-Dimension). Warum sind solche Urteile dennoch möglich?

In diesem Zusammenhang ist hilfreich, sich zu vergegenwärtigen, wie in der Politikwissenschaft über die „Integration durch Recht" gedacht wird, genauer: in jenem einflussreichen Teil der Integrationsforschung, der sogenannte ,intergouvernementale" Erklärungen für den Verlauf des Integrationsprozesses bereithält. Autoren wie Moravcsik (1998) und Garrett (1992; 1995) sehen die Mitgliedstaaten weiter als Träger und Kontrolleure des Integrationsprozesses. Gegenüber der Annahme, supranationale Agenturen wie der EuGH könnten sich auf Dauer von den Präferenzen der Mitgliedstaaten entfernen, verhalten sich Vertreter dieser Perspektive skeptisch. Denn der unbestrittene Nachweis, dass der EuGH systematisch über den Status quo der Verträge hinausgeht und das europäische Recht zuungunsten nationalen Rechts ausweitet, ist - so diese Sicht nicht als Nachweis einer Entfernung von den „wahren“ Präferenzen der Mitgliedstaaten und der dominanten Akteure in ihnen zu verstehen. Der EuGH verfügt über keine eigenen Vollzugsorgane und ist darauf angewiesen, dass die Mitgliedstaaten das von ihm gesetzte Recht umsetzen. Nichtumsetzung bleibt stets möglich, und um einen derartigen Konflikt nicht entstehen zu lassen, bleibt dem EuGH nur, den „Präferenzraum“ der Mitgliedstaaten nicht zu verlassen. Anders gewendet: Der Akt der Umsetzung wird als politisch-materielle Zustimmung zur Integrationspolitik des
EuGH gewertet. Die politische Legitimierung richterlicher Rechtsfortbildung erfolgt über den Umsetzungsakt, der alles andere als selbstverständlich ist, weil Akte der Nichtanwendung als ebenso möglich wie prinzipiell legitim erscheinen.

Denkt man die jüngste Urteilsserie aus dieser Perspektive durch, dann stellt sich die Frage, warum sich die Rechtschöpfungspraxis des EuGH nicht deutlicherer Kritik ausgesetzt sieht. Wäre nicht zu erwarten, dass die Legitimität europäischer Übergriffe auf das nationale Arbeitskampfrecht grundsätzlich in Zweifel gezogen wird? Und dass insbesondere die Gewerkschaften offensiv die nationalen Arbeitsund Sozialverfassungen vor transformativen Zugriffen der europäischen Ebene schützen? Gewiss, die gewerkschaftlichen Stellungnahmen $\mathrm{zu}$ den Urteilen waren deutlich und nicht von einer, wie Hans-Jürgen Urban (2009) sagt, „naiven Europafreundlichkeit" geprägt. Gleichzeitig aber fällt auf, dass die zentrale Forderung nach europäischen sozialen Grundrechten (Abschnitt 5) vor allem von der Hoffnung auf eine dann veränderte Rechtsprechungspraxis des EuGH getragen ist, weniger hingegen von einer grundsätzlichen Infragestellung der Legitimität der „Integrationspolitik durch Recht". Fragt man, warum das so ist, wären meinem Eindruck nach insbesondere drei Gesichtspunkte zu bedenken:

(1) Die europäische Integration als „Ersatzideal": Über einen langen Zeitraum wurde die europäische Integration von einem aus guten historischen Gründen unerschütterlichen außenpolitischen Konsens getragen. Die deutsche Linke war stets nicht nur Teilnehmerin, sondern besondere Verfechterin dieses Konsenses, was in den internationalistischen Grundüberzeugungen der Arbeiterbewegung wurzelt. Autoren wie $A n-$ ne-Cécile Robert (Le Monde Diplomatique 13.5.2005, S. 4f.) haben die These aufgestellt, dass die europäische Integration nach dem weitgehenden Verlust sozialistischer Ideale als „Ersatzideal“ linker Politik fungiert. Spätestens mit der Einheitlichen Europäischen Akte und dem MaastrichtProgramm aber ist europäische Politik von der zwischenstaatlichen Politik zu einem festen Bestandteil der Innenpolitik geworden und bedarf anderer Antworten als des Verweises auf einen außenpolitischen Konsens. Meine These ist, dass die europapolitische Programmatik Pfadabhängigkeiten aufweist und nur langsam von außen- auf innenpolitische Prüfkriterien umschaltet.

(2) Sequenzen der Rechtsfortbildung: Der in diesem Beitrag diskutierten Urteilsserie geht eine Sequenz von EuGH-Urteilen voraus, die auf Grundlage vergleichsweise schmaler Bestimmungen in den europäischen Verträgen - vor allem: dem Grundsatz der Lohngleichheit der Geschlechter und der Arbeitnehmerfreizügigkeit - einen wachsenden europäischen Bestand an Antidiskriminierungsregeln schuf. Diese Urteile sind nicht mit Sozialpolitik zu verwechseln, tragen sie doch vor allem marktbefähigende Züge (im Sinne von: freie, aber faire Märkte; ausführlich hierzu: Somek 2008). ${ }^{9}$ Die deutschen Gewerkschaften haben diese Urteile stets begrüßt und damit die durch Richterrecht geschaffene Drittwirkung des Europarechts auf Private bejaht, ungeachtet gewagter Rechtskonstruktionen, wie sie jüngst beispielsweise im Urteil zum Fall Mangold (C-144/04 vom 22.11.2005) zum Ausdruck kamen. Die hier erfolgte grundsätzliche Akzeptanz der Drittwirkung des Europarechts auf Private erschwert grundsätzliche Kritik in einer Judikatur, in der sich die Verpflichtung Privater nunmehr gegen Gewerkschaften richtet.

(3) Heterogene Interessen der Gewerkschaftsverbände: Die europäischen Teilorganisationen der Gewerkschaften haben im Zuge der Wirtschaftsintegration zunehmend eigene Zuständigkeitsbereiche, organisationale Eigeninteressen und ein entsprechendes Eigenleben entwickelt. Die Radikalisierung der Binnenmarktintegration berührt zwei sich überlagernde Konfliktlinien: Marktliberalisierung gegenüber sozial eingebettetem Kapitalismus einerseits, supranationale gegenüber nationalen Gestaltungskompetenzen andererseits. Antworten auf Probleme der europäischen Integration werden zuvorderst von den europäischen Verbänden formuliert, die an Relevanz gewinnen, wenn die Integration voranschreitet. Nur vor diesem Hintergrund ist zu ver-

\footnotetext{
9 Bei einigen dieser Urteile allerdings ging es im engeren Sinne um Sozialpolitik: um Beseitigung von Restriktionen des transnationalen Zugangs zu den sozialen Sicherungssystemen der Mitgliedstaaten. Vgl. hierzu die ausführliche Diskussion bei Scharpf (2008b, S. 90ff.), der als Folge dieser Rechtsprechung vor allem Deregulierungsdruck auf die skandinavischen und kontinentaleuropäischen Wohlfahrtsstaaten erwartet.
} 
stehen, warum der EGB in seiner ersten Stellungnahme zum Laval-Urteil vor der "Gefahr" von Bestrebungen nationaler Gewerkschaften warnen konnte, ihre jeweiligen Sozialsysteme vor europäischem $\mathrm{Zu}$ griff zu schützen - und dies, wie in Abschnitt 1 zitiert, mit „protektionistischen Reaktionen“identifizierte.

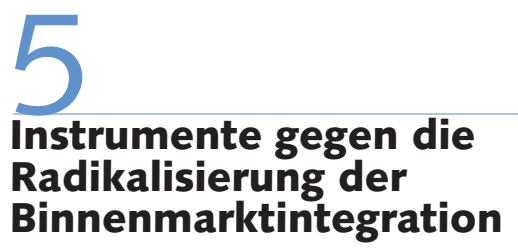

Liegt die Lösung des oben herausgearbeiteten Problems nicht offensichtlich bereit: Die Ratifizierung des vorläufig gescheiterten EU-Reformvertrags, mit dem die Grundrechtecharta auf Vertragsebene und damit auf Augenhöhe mit den Grundfreiheiten gehoben würde? Sind die Urteile zu Viking, Laval, Rüffert und Kommission gegen Luxemburg nicht die konsequente Folge einer asymmetrischen Normenhierarchie, die Grundfreiheiten über Grundrechte stellt? Diese Interpretation übersieht, dass der EuGH schon lange, im Rahmen eines weiteren Akts richterlicher Rechtschöpfung, mit europäischen Grundrechten als „allgemeinen Rechtsgrundsätzen der Gemeinschaft" operiert und diese wie andere Bestandteile des Primärrechts behandelt, ohne dass dies der Abwägung dieser Grundrechte gegen die Grundfreiheiten im Wege stehen würde. Wohl gemerkt: Nach gegebenem Stand der Dinge wäre ein verbindlicher, kodifizierter EU-Grundrechtskatalog zweifellos zu begrüßen. Er wäre allerdings, so mein Argument, kein geeignetes Instrument zur Abwehr der Radikalisierung der Binnenmarktintegration.

\subsection{EUROPÄISCHE SOZIALE GRUNDRECHTE ALS HEBEL?}

Niemand käme auf die Idee, einem zwischenstaatlichen Abkommen zur Förderung der wirtschaftlichen Zusammenarbeit einen Grundrechtskatalog beizufügen (Weiler 1986, S. 1110). Dessen Fehlen markiert somit kein Versäumnis der Gründungsväter der Europäischen Wirtschaftsgemeinschaft der 1950er Jahre. So hatte der EuGH die Existenz spezifisch europäischer Grundrechte auch zunächst verneint. Diese Haltung änderte sich seit den späten 1960er Jahren (Haltern 2005, Kapitel 6). In einem Urteil aus dem Jahr 1969 (Stauder, C-29/69 vom 12.11.1969) erwähnte der EuGH erstmals die „in den allgemeinen Rechtsgrundsätzen der Gemeinschaftsrechtsordnung, deren Wahrung der Gerichtshof $\mathrm{zu}$ sichern hat, enthaltenen Grundrechte der Person“ (Rn. 7). Damit signalisierte der EuGH, dass er sich dazu berufen fühlte, einen europäischen Grundrechtskatalog judikativ zu entwickeln. Fünf Jahre später, in seinem Urteil zur Rechtssache Nold (C-4/73 vom 14.5. 1974), äuBerte sich das Gericht zu den Rechtsquellen dieser Grundsätze, die einerseits in dem Substrat der nationalen Verfassungsüberlieferungen, andererseits in der Europäischen Menschenrechtskonvention bestanden. Fortan erkannte der EuGH diverse Einzelgrundrechte als Bestandteile allgemeiner Rechtsgrundsätze an, so beispielsweise die Menschenwürde (Casagrande, C-9/74 vom 3.7.1974), die Religions- und Bekenntnisfreiheit (Prais, C-415/93 vom 15.12.1995) und die Unverletzlichkeit der Wohnung (Hoechst, C-46/87 vom 21.9. 1989).

Entscheidend sind nun die Gründe, die den EuGH bewogen, Grundrechte auch soziale Grundrechte - als allgemeine Rechtsgrundsätze $\mathrm{zu}$ entwickeln und in das europäische Primärrecht einzuordnen. Vergegenwärtigen wir uns noch einmal die Situation, die durch die Rechtschöpfungsakte der 1950er und 1960er Jahre entstanden war. Der EuGH hatte die Grundsätze des Vorrangs und der unmittelbaren Wirkung europäischen Primär- und Sekundärrechts vor mitgliedstaatlichem Recht entwickelt. Da das Gericht hierbei nicht zwischen Verfassungs- und Gesetzesrecht unterschied, musste jede europäische Richtlinie im Kollisionsfall nationales Grundrecht ausschalten. Dass dies aus Sicht der nationalen Verfassungsgerichte nicht akzeptabel war, lag auf der Hand. Auf diese Situation reagierte das deutsche Bundesverfassungsgericht (BVerfG) mit seiner sogenannten Solange-I-Entscheidung, mit dem es sich in direkten Widerspruch zur vom EuGH entwickelten Vorrangdoktrin stellte und sich vorbehielt, die Vereinbarkeit von europäischem Recht mit deutschem Verfassungsrecht in jedem Einzelfall zu prüfen (Bröhmer 1999, S. 31).

Das konnte in der Konsequenz nur bedeuten, dass sich die Vorrangdoktrin auf jene eng umrissenen Bereiche beschränken musste, die nationale Grundrechtsausübung nicht tangierte. Wirkte europäisches
Recht aber mittelbar in mitgliedstaatliche Grundrechtsausübung hinein, musste die Vorrangdoktrin unweigerlich Widerstand der nationalen Verfassungsgerichte hervorrufen und konnte deshalb nur eingeschränkte Wirkung entfalten. Die Konstruktion europäischer Grundrechte als allgemeine Rechtsgrundsätze war somit vor allem ein strategisches Instrument zur Verteidigung der Expansion europäischen Rechts gegenüber nationalem Recht. Denn nun konnte der EuGH aus seiner Sicht notwenige Abwägungen zwischen Grundfreiheiten und Grundrechten selbst vornehmen - und die Ausübung Letzterer im Zweifel auch, wenn er es für richtig hielt, zugunsten Ersterer einschränken. Wie wir gesehen haben, ist die Bereitschaft, hierbei über eine bloße Missbrauchskontrolle hinauszugehen, die eigentliche Neuerung der mit Viking, vor allem aber Laval begonnenen Urteilsserie. ${ }^{10}$

Die Hoffnung, die Rechtsprechungspraxis des EuGH werde durch die Verankerung sozialer Grundrechte im Vertragswerk in eine neue Richtung gelenkt, erscheint somit fragwürdig. Die vom EuGH als allgemeine Rechtsgrundsätze anerkannten Grundrechte rangieren bereits auf derselben logischen Ebene wie die Grundfreiheiten. In ihren Reaktionen auf die jüngste Urteilsserie warfen die Gewerkschaften dem EuGH vor, sozialen Grundrechten im Vergleich zu den Grundfreiheiten eine zu niedrige Geltung einzuräumen (vgl. http:// www.etuc.org/IMG/pdf_ResolutionDE-2. pdf. S. 3) und forderten, soziale Grundrechte im Kollisionsfall stärker zu gewichten als die Grundfreiheiten (ebd. S. 5; Michael Sommer im einblick 18 vom 20.10. 2008, S. 7). ${ }^{11}$ Würde das Eingriffe in die Grundrechtsausübung im Namen der Grundfreiheiten verhindern? An dieser Stelle scheint mir ein grundsätzlicher Denkfehler zu bestehen. Nehmen wir an, der EuGH würde die europäischen Grund-

\footnotetext{
10 Diese Einschätzung zum Hintergrund der Konstruktion europäischer Grundrechte durch den EuGH ist nicht zynisch, sondern beschreibt gesicherten Forschungsstand. "The prime motivation for judicial extension was not ... simply a benevolent interest in human rights... The, surface language' of the Court in Stauder and its progeny is the language of human rights. The 'deep structure' is all about supremacy", so Weiler (1986, S. 1118ff.).

11 Ich vernachlässige hier, dass diese Forderung wegen des Erfordernisses der Einstimmigkeit bei Vertragsänderungen im Europa der 27 nicht durchsetzbar erscheint.
} 
freiheiten in künftigen Zweifelsfällen mit nur $10 \%$, soziale Grundrechte hingegen mit $90 \%$ gewichten. Das würde nichts daran ändern, dass nationales Arbeits- und Sozialrecht, das in allen Mitgliedstaaten bereits einen historischen Kompromiss zwischen den Erfordernissen des Markts und dem politischen Willen zu marktkorrigierenden Maßnahmen reflektiert, vom EuGH im Zuge der Marktintegration erneut gegen die Erfordernisse des Markts, nunmehr in Gestalt der europäischen Grundfreiheiten abgewogen wird - mit welchem Gewichtungsverhältnis auch immer. Eine primärrechtliche Verankerung der Grundrechte würde nichts daran ändern, dass ihre Ausübung in der Auslegung des EuGH nur unter Achtung der Binnenmarktregeln erfolgen darf. ${ }^{12}$

\subsection{POLITISCH GEWOLLTE GRENZEN DER INTEGRATION VERTEIDIGEN}

Das Problem wird also nicht durch die Aufnahme sozialer Grundrechte in die Verträge gelöst, so elegant dieser genuin „europäische" Weg auch erscheinen mag. Die Lösung kann nur in einer von den Mitgliedstaaten politisch überwachten Selbstzurückhaltung des EuGH bestehen, mit Rechtsnormen wie dem Streikrecht der EU-Mitgliedsländer autonomieschonend umzugehen, den Arbeits- und Sozialsystemen der Mitgliedstaaten also jene umfassende Bereichsautonomie zuzugestehen, die mit Art. 137 Abs. 5 EGV von den vertragschließenden Staaten politisch gewollt war. Politisch überwachte Selbstzurückhaltung vor Überinterpretation der europäischen Grundfreiheiten und deren Anwendung auf nationales Arbeits- und Sozialrecht setzt Willen und Fähigkeit der Mitgliedstaaten voraus, diese Überwachung tatsächlich durchzuführen und den Vorrang europäischen Rechts vor nationalem Recht in Zweifel zu ziehen, wenn mit ihm transformativ auf Gegenstände zugegriffen werden soll, die dem demokratischen Selbstbestimmungsrecht der Mitgliedstaaten obliegen und nicht durch einen politischen Akt an die europäische Ebene delegiert wurden.

Für die betroffenen Verbände hat die Forderung nach europäischen sozialen Grundrechten den Vorzug, bekanntes Fahrwasser nicht verlassen zu müssen, nämlich: auf Probleme der europäischen Integration mit der Forderung nach mehr Integration $\mathrm{zu}$ antworten. ${ }^{13}$ Diese Strategie, so meine ich, ist nunmehr an Grenzen gestoßen. Politisch überwachte Selbstzurückhaltung des EuGH bedeutet, Grenzen der Wirtschaftsintegration zu definieren. Solche Grenzen müssen freilich nicht auf alle Ewigkeit bestehen. Die Hoffnungen bleiben an die europäische Politik gerichtet, irgendwann so handlungsfähig zu werden, dass sie der Integrationspolitik des EuGH auf Augenhöhe gegenübertreten kann. Dieses Ziel ist, wohl gemerkt, ein Fernziel. ${ }^{14}$ Der weitere Verlauf der europäischen Integration ist offen und kann von eher europa-optimistischen Denkern ebenso wenig vorhergesagt werden wie von Euroskeptikern. Solange aber die Europäische Union nicht über die politischen Mittel verfügt, unionsweite Tarifvertragssysteme, Arbeitsrechte und Sozialsysteme selbst herzustellen und ihre Stabilität zu gewährleisten, kann die Bewahrung des sozial eingebetteten Kapitalismus nur erreicht werden, wenn die Autonomie von Regelungsbeständen, die der europäischen Integration aus guten Gründen nicht anheim gestellt wurden, gewahrt und im Konfliktfall verteidigt wird.

\section{Ausblick}

Die EuGH-Urteile zu Viking, Laval, Rüffert und Kommission gegen Luxemburg haben in Parteien, Verbänden und Verwaltung große Irritationen hervorgerufen. Im vorliegenden Diskussionsbeitrag habe ich in Ergänzung zur juristischen Auseinandersetzung mit den Urteilen eine historischinstitutionalistische Perspektive auf den EuGH und seine jüngsten Grundsatzentscheidungen vorgeschlagen. Ich habe die jüngste Urteilsserie in den historischen Kontext der Rechtsfortbildung durch den EuGH gestellt und aufgezeigt, dass es dem Gericht seit den 1960er Jahren gelang, seinen Einflussbereich gegenüber nationalem Recht durch die sukzessive Aneignung von Regelungsbefugnissen zu erweitern. Daraus resultiert mein Vorschlag, die vier Urteile nicht als „Unfälle“ unsauber agierender Richter oder als „Anomalien“ der europäischen Integration, sondern als im historischen Kontext konsequenten Versuch der Einleitung einer neuen Phase der Binnenmarktintegration zu verstehen (vgl. zur These einer neuartigen Phase der Binnenmarktintegration auch Höpner/Schäfer 2008).
Die vier Urteile zehren, wie ich im Einzelnen gezeigt habe (Abschnitt 2), von Grundlagen, die ihrerseits auf dem Wege richterlicher Rechtsfortbildung entstanden. Inhaltlich besteht die Radikalisierung der Rechtsprechung darin, dass die Prüfung arbeitsmarkt- und sozialpolitischer Regulierung an den europäischen Grundfreiheiten von einer de facto autonomieschonenden Missbrauchskontrolle auf gezielte institutionelle Transformation umschaltet (Rödl 2009, S. 888ff.). Der Gestaltungsspielraum der Mitgliedstaaten, eigene Lösungen für den Ausgleich zwischen kapitalistischer Ökonomie und sozialer Demokratie zu finden, schmilzt damit auf jenes Minimum zusammen, das die EuGHRichter als von zwingenden Gründen des Allgemeininteresses getragen sowie als verhältnismäßig akzeptieren (Abschnitt 3).

Die Urteile sind somit keine Fehlentscheidungen oder Anomalien, sondern ein weiterer Schritt in einer historischen Abfolge der Usurpation von Zuständigkeiten. Sie markieren ein gewagtes strategisches Experiment - und sollten als solches auch behandelt und verarbeitet werden. Sie zielen auf Fortentwicklung der Integration, sind aber faktisch eine Kampfansage an die Existenz autonomer nationalstaatlicher Arbeits- und Sozialverfassungen, deren Regulierungsniveau über ein vom EuGH definiertes Mindestmaß hinausgeht. Bleiben die Urteile zu Viking, Laval, Rüffert und Kommission gegen Luxemburg unwider-

12 Aus diesem Grund halte ich die positive Würdigung des Viking-Urteils durch den EGB - weil der EuGH darin das Streikrecht als allgemeinen Rechtsgrundsatz der Gemeinschaft anerkannt habe - für ebenso unangemessen wie verblüffend. Was ist damit gewonnen, wenn das Gericht das Streikrecht in Abwägungsfragen nicht anders behandelt als jede andere (vermeintliche) Störung des Binnenmarkts? Und wie hätte ein Urteil, das die Existenz eines Streikrechts verneint, eigentlich aussehen sollen?

13 Anders gewendet: Die Forderung nach sozialen Grundrechten im europäischen Primärrecht ist bemüht, das diskutierte Problem als Problem der horizontalen Gewaltenteilung auf europäischer Ebene zu behandeln, nicht aber als Problem der vertikalen Gewaltenteilung zwischen europäischer Ebene und den Mitgliedstaaten.

14 Die Erweiterung der EU auf 27, bald vielleicht 29 Mitgliedstaaten lässt dieses Ziel in weitere Ferne rücken und verschärft damit die Asymmetrie von negativer und positiver Integration. Denn die mit den Erweiterungsrunden zunehmende Heterogenität der Interessen erschwert sowohl koordinierten Widerstand gegen Überdehnungen der Grundfreiheiten durch den EuGH als auch die Verständigung auf Projekte der positiven, marktkorrigierenden Integration. 
sprochen, verfügt das Europarecht nunmehr über ein Instrumentarium, mit dem sich die nationalen Eigenständigkeiten der sozialen Regulierung des Kapitalismus im Prinzip Stück für Stück aushebeln lassen. Angesichts dieses Instrumentariums geraten marktkorrigierende Regulierungen in die institutionalisierte Defensive. Sie müssen ihren Bestand vor dem EuGH in jedem Einzelfall als objektiv zwingend erforderlich und als dieses erforderliche Maß nicht überschreitend rechtfertigen. Das gilt für alle Formen der Regulierung, die potenziell mit europäischen Grundfreiheiten kollidieren - und damit tendenziell tatsächlich für die gesamten Arbeits- und Sozialverfassungen, dienen sie doch keinem anderen Zweck, als kapitalistisches Marktverhalten $\mathrm{zu}$ transzendieren und es in sozialverträgliche Bahnen zu lenken.

Der Ausgang dieses Experiments ist offen. Intergouvernmentalisten wie Moravcsik (1998) hatten stets argumentiert, dass sich die Akzeptanz - und deshalb, mittelbar, auch die Legitimität - der Kompetenzaneignungen des EuGH daraus ablei- ten lässt, dass seine Urteile befolgt und von den nationalen Rechtssystemen verarbeitet werden. Nichtanwendung, so diese Sicht, bleibt stets möglich (ebd., Abschnitt 4). Ihre Wahrscheinlichkeit ist schwer zu bestimmen. Einerseits sind die Hürden dafür hoch. Andererseits aber sollte die Fragilität der materiellen Steuerungsfähigkeit des EuGH nicht unterschätzt werden. Politikwissenschaftler wie Caldeira und Gibson (1995) haben nachgewiesen, dass sich der EuGH im Konfliktfall auf kein hohes Maß an diffuser öffentlicher Unterstützung würde verlassen können (ebd., S. 357ff., 372). Und die Wahrscheinlichkeit politischer Gegenreaktionen steigt mit dem Ausmaß an Irritationen, die der EuGH in den politischen Systemen der Mitgliedstaaten auslöst.

Mein Vorschlag lautet, angesichts der Radikalität der vom EuGH eingeleiteten Weichenstellungen nicht zur Tagesordnung überzugehen und die dringend nötige kritische Diskussion nicht an die Juristen und Europapolitiker zu delegieren. In den betroffenen Verbänden scheint man sich auf die Forderung nach der Verankerung sozialer Grundrechte in den europäischen Verträgen, verbunden mit einer etwaigen Höhergewichtung gegenüber den europäischen Marktfreiheiten, verständigt zu haben. So sympathisch diese Forderung im Hinblick auf das Soziale Europa erscheinen mag - sie wird, wie ich im Einzelnen dargelegt habe (Abschnitt 5), die Bestände der sozialen Demokratie nicht aus ihrer institutionalisierten Defensive befreien. Die Lösung kann nur, so habe ich argumentiert, in einer politisch überwachten und kontrollierten, die Autonomie der mitgliedstaatlichen Arbeits- und Sozialverfassungen schonenden Selbstzurückhaltung des EuGH vor Überinterpretationen der in den Römischen Verträgen von 1957 verankerten Marktfreiheiten liegen. Diese Strategie steht und fällt mit dem politischen Willen und der strategischen Fähigkeit zur politischen Kontrolle gegenwärtiger und zukünftiger „Integration durch Recht“. 


\section{LITERATUR}

Bröhmer, J. (1999): Das Bundesverfassungsgericht und sein Verhältnis zum Gerichtshof der Europäischen Gemeinschaften, in: Aus Politik und Zeitgeschichte 16, S. 31-39

Büchele, M. (2008): Diskriminierung, Beschränkung und Keck-Mithouard - die Warenverkehrsfreiheit, in: Roth, G. H./Hilpold, P. (Hrsg.): Der EuGH und die Souveränität der Mitgliedstaaten. Eine kritische Analyse richterlicher Rechtsschöpfung auf ausgewählten Rechtsgebieten, Wien, S. 335394

Caldeira, G. A./Gibson, J. L. (1995): The Legitimacy of the Court of Justice in the European Union: Models of Institutional Support, in: American Political Science Review 2, S. 356-376

Frenz, W. (2004): Handbuch Europa-Recht. Band 1: Europäische Grundfreiheiten, Berlin/Heidelberg

Garrett, G. (1992): International Cooperation and Institutional Choice: The European Community's Internal Market, in: International Organization 2, S. 533-560

Garrett, G. (1995): The Politics of Legal Integration in the European Union, in: International Organization 1, S. 171-181

Haltern, U. (2005): Europarecht und das Politische, Tübingen

Hitzel-Cassagnes, T. (2004): Geltung und Funktion. Supranationale Gerichtsbarkeit im Spannungsfeld von Praktischer Rationalität, Recht und Demokratie, Baden-Baden

Höpner, M. (2008): Usurpation statt Delegation. Wie der EuGH die Binnenmarktintegration radikalisiert und warum er politischer Kontrolle bedarf, Max-Planck-Institut für Gesellschaftsforschung, Discussion Paper 08/12, Köln

Höpner, M./Schäfer, A. (2008): Eine neue Phase der europäischen Integration: Legitimitätsdefizite europäischer Liberalisierungspolitik, in: Höpner, M./Schäfer, A. (Hrsg.): Die Politische Ökonomie der europäischen Integration, Frankfurt/New York, S. 129-156
Moravcsik, A. (1998): The Choice for Europe. Social Purpose and State Power from Messina to Maastricht, Ithaca

Rasmussen, H. (1986): On Law and Policy in the European Court of Justice, Dordrecht u.a.

Rödl, F. (2009): Arbeitsverfassung, in: von Bogdandy, A. (Hrsg.): Europäisches Verfassungsrecht, Berlin u.a., S. 855-904

Scharpf, F. W. (2008a): „Der einzige Weg ist, dem EuGH nicht zu folgen“ (Interview), in: Mitbestimmung 7/8, S. 18-23

Scharpf, F. W. (2008b): Individualrechte gegen nationale Solidarität, in: Höpner, M./Schäfer, A. (Hrsg.): Die Politische Ökonomie der europäischen Integration, Frankfurt/New York, S. 89-99

Schmidt, S. K. (200)0: Only an Agenda Setter? The European Commission's Power over the Council of Ministers, in: European Union Politics 1, S. 37-61

Somek, A. (2008): Das europäische Sozialmodell: Diskriminierungsschutz und Wettbewerb, in: juridikum 3, S. 118-125

Streeck, W./Thelen, K. (2005): Introduction: Institutional Change in Advanced Political Economies, in: Dies. (Hrsg.): Beyond Continuity. Institutional Change in Advanced Political Economies, New York, S. 1-39

Urban, H.-J. (2009): Den EU-Prozess neu programmieren. Was Gewerkschaften dem Europa der Eliten entgegensetzen, in: Mitbestimmung 3, S. 28-32

Vauchez, A. (2007): Embedded Law - Political Sociology of the European Community of Law: Elements of a Renewed Research Agenda. Robert Schuman Centre for Advanced Studies, Working Paper 23, San Domenico di Fiesole

Weiler, J. H. H. (1986): Eurocracy and Distrust: Some Questions Concerning the Role of the European Court of Justice in the Protection of Fundamental Human Rights within the Legal Order of the European Communities, in: Washington Law Review 3, S. 1103-1142 